\title{
Neuroprotective effects of dynamic bubble trap use in patients undergoing pulmonary endarterectomy: a two-arm randomized controlled trial
}

\author{
Jasmin El Shazly ${ }^{1,2}$, Tibo Gerriets ${ }^{1,3,4}$, Juergen Hennig ${ }^{5}$, Marius Butz ${ }^{1,3}$, Sabrina Kastaun ${ }^{6}$, \\ Christoph B. Wiedenroth $^{7}$, Markus Schoenburg ${ }^{1,8}$, Marc Wollenschlaeger ${ }^{9}$, Georg Bachmann ${ }^{1,10}$, \\ Stefan Guth $^{7 \#}$, Martin Juenemann ${ }^{1,3 \#}$
}

${ }^{1}$ Heart and Brain Research Group, Kerckhoff Heart and Lung Center, Bad Nauheim, Germany; ${ }^{2}$ Department of Psychocardiology, Kerckhoff Heart and Lung Center, Bad Nauheim, Germany; ${ }^{3}$ Department of Neurology, University of Giessen, Giessen, Germany; ${ }^{4}$ Department of Neurology, Health Center Wetterau, Friedberg, Germany; ${ }^{5}$ Division of Personality Psychology and Individual Differences, University of Giessen, Giessen, Germany; ${ }^{6}$ Institute of General Practice, Addiction Research and Clinical Epidemiology Unit, Medical Faculty of the Heinrich-Heine-University Düsseldorf, Düsseldorf, Germany; ${ }^{7}$ Department of Thoracic Surgery, Kerckhoff Heart and Lung Center, Bad Nauheim, Germany; ${ }^{8}$ Department of Cardiac Surgery, Kerckhoff Heart and Lung Center, Bad Nauheim, Germany; ${ }^{9}$ Perfusion Service, Kerckhoff Heart and Lung Center, Bad Nauheim, Germany; ${ }^{10}$ Department of Radiology, Kerckhoff Heart and Lung Center, Bad Nauheim, Germany

Contributions: (I) Conception and design: J El Shazly, T Gerriets, M Butz, S Kastaun, M Schoenburg, M Wollenschlaeger, G Bachmann, S Guth, M Juenemann; (II) Administrative support: T Gerriets, S Guth, M Schoenburg; (III) Provision of study materials or patients: J El Shazly, M Butz, M Juenemann; (IV) Collection and assembly of data: J El Shazly, S Kastaun, M Butz; (V) Data analysis and interpretation: J El Shazly, M Butz; (VI) Manuscript writing: All authors; (VII) Final approval of manuscript: All authors.

\#These authors contributed equally to this work.

Correspondence to: Jasmin El Shazly, MSc. Department of Psychocardiology, Kerckhoff Heart and Lung Center, Benekestraße 2-8, 61231 Bad Nauheim, Germany. Email: jasmin.el-shazly@psychol.uni-giessen.de.

Background: During cardiosurgical procedures that use extracorporeal circulation (ECC), a variety of neurological complications can occur, and postoperative cognitive deficits remain an unsolved problem. Among the sources of these complications are intraoperatively detectable cerebral microemboli, which mainly consist of air. This study's purpose was to assess neuroprotective effects of reducing these gaseous microemboli using a dynamic bubble trap (DBT) in patients undergoing pulmonary endarterectomy (PEA) for the treatment of chronic thromboembolic pulmonary hypertension (CTEPH).

Methods: Patients undergoing PEA were randomly assigned to receive either a DBT (n=47) or no additional device (controls, $\mathrm{n}=46$ ) during ECC. Neuropsychological testing was performed before and 3 months after PEA. The primary endpoint was cognitive improvement in the DBT group (n=29) compared with the control group $(n=42)$. As secondary endpoint, ischemic brain micro-lesions were analyzed on postoperative days 6 through 10 using diffusion-weighted magnetic resonance imaging (MRI).

Results: Analysis of interaction effects revealed improved performance in visual long-term memory $\left(\mathrm{P}=0.008, \eta^{2}=0.099\right)$, verbal long-term memory $\left(\mathrm{P}=0.030, \eta^{2}=0.067\right)$, verbal short-term memory $(\mathrm{P}=0.014$, $\left.\eta^{2}=0.083\right)$, and attention and processing speed $\left(\mathrm{P}=0.043, \eta^{2}=0.056\right)$ from pre- to post-testing in the DBT group compared to control group. In MRI, postoperative ischemic micro-lesions could only be detected in one patient; another patient suffered a severe bihemispheric embolic stroke.

Conclusions: DBT positively influences memory function after PEA. This effect is most likely caused by the reduction of gaseous microemboli.

Trial Registration: This study is registered in the German Clinical Trials Register, ID: DRKS00021499. 


\begin{abstract}
Keywords: Pulmonary endarterectomy (PEA); neuroprotection; neuropsychological function; postoperative cognitive function
\end{abstract}

Submitted May 14, 2021. Accepted for publication Jul 30, 2021.

doi: $10.21037 /$ jtd-21-831

View this article at: https://dx.doi.org/10.21037/jtd-21-831

\section{Introduction}

Chronic thromboembolic pulmonary hypertension $(\mathrm{CTEPH})$ is a rare disease of the pulmonary vasculature and a specific type of pulmonary hypertension (1-3) characterized by obstruction or occlusion of subsegmental, segmental or larger pulmonary arteries by the fibrotic residuals of pulmonary embolism (4). Removing the obstructing material by performing a pulmonary endarterectomy (PEA) is the gold standard for operable CTEPH. It should be noted, however, that in many patients with CTEPH, mean pulmonary pressure (mPAP) at rest can be normalized by surgical or multimodality treatment, and patients feel healthy, but it is unlikely that all pulmonary vessels will return to normal (5). The goal of PEA surgery is that patients with reduced PAP recover to achieve improved functional status and quality of life (6). Therefore, PEA is the most effective treatment for CTEPH (7). To achieve the goal of removing the obstructing material, extracorporeal circulation (ECC) with several episodes of circulatory arrest is necessary to obtain a blood-free surgical area, and deep hypothermia is required to reduce metabolic activity and protect organ function (8).

Few studies address neuropsychological outcomes after PEA. In the PEACOG study, cognitive function improved 3 months after PEA (9). Another study by Vanini et al. found a post-surgical improvement in motor speed, which was accompanied by improved quality of life and reduced symptoms of depression and anxiety (8). Such favorable neuropsychological changes may be attributed to improved cardiac output (9) and enhanced post-surgical hemodynamics (8).

Previous studies reported associations between the use of ECC during surgical procedures and the presence of various neurological complications $(10,11)$. These side effects can be partly attributed to intraoperatively detectable cerebral microemboli, of which the majority appear to consist of air (12). In this context, scientific evidence mainly refers to cardiosurgical procedures: Although severe neurological complications such as brain infarctions or global ischemia have become rare with the use of ECC, postoperative cognitive decline (POCD) remains an unsolved problem (13-15). The pathophysiology of POCD remains the subject of controversial debate, but the consequences of cognitive impairment are well described. Besides a decline in health-related quality of life (HQL) $(16,17)$, POCD can contribute to premature retirement, as well as increased social dependency (18). Data from cardiac surgery suggest that the use of a dynamic bubble trap (DBT) in the arterial line of the heart-lung machine significantly reduces the intraoperative embolization rate, yielding a significant improvement in cognitive performance at three months following surgery (11). Patients undergoing PEA may be exposed to an increased level of gaseous embolism due to proportionally long perfusion times at the ECC. Therefore, these patients should benefit notably from a filter system that can eliminate a large part of the gaseous emboli from the arterial circulation, resulting in better neuropsychological test performance. Following the results of other studies $(8,9)$, patients undergoing PEA are not expected to show cognitive deterioration. Accordingly, it is assumed that the neuropsychological performance of patients receiving a DBT will experience greater improvement compared to patients who receive the standard ECC procedure without the use of a DBT. The aim of this two-arm randomized controlled trial was to evaluate the neuroprotective effect of reducing gaseous microemboli using a DBT in patients undergoing PEA for the treatment of CTEPH.

We present the following article in accordance with the CONSORT reporting checklist (available at https://dx.doi. org/10.21037/jtd-21-831).

\section{Methods}

\section{Patient enrollment and study design}

This study was designed as a prospective, double-blind, single-center, two-arm randomized controlled trial. Between April 2012 and March 2018, 764 PEA surgeries were 
performed in this center, with many patients ineligible for the study due to the distance of the clinic from the place of residence. One hundred patients scheduled for PEA surgery at the Kerckhoff Clinic in Bad Nauheim were prospectively enrolled in this study and randomized into two groups. Patients in the DBT group received a DBT (Kardialgut, Petersdorf, Germany) placed in the arterial line of the ECC between the arterial filter and the arterial cannula. Patients in the control group received the standard ECC procedure without the use of an additional device. Randomization was implemented using a computer-generated list with an 1:1 allocation ratio. The randomization list was generated by the study coordinator prior to the first patient in. A member of the thoracic surgery secretariat managed the list and informed the perfusionist about the group allocation for each patient. This person was not involved in any other processes of the study.

Patients and all investigators were blinded to group assignment. Eligible patients were all adults aged $\geq 18$ years and scheduled for an elective PEA. Because neuropsychological testing is language-dependent, another inclusion criterion was that German be the participants' native language. Exclusion criteria were pre-existing neurological or psychiatric diseases (cerebral infarction, craniocerebral trauma, dementia or manifest depression) that might influence neuropsychological testing. Only patients living within $300 \mathrm{~km}$ of the clinic were selected for the study. For the follow-up testing, the effort to travel to the clinic was otherwise considered too strenuous.

The present study complies with the Declaration of Helsinki (as revised in 2013) and was approved by the ethics committee of Justus-Liebig University Giessen (AZ 157/11). Written informed consent was obtained from all participants prior to study inclusion. This study is registered in the German Clinical Trials Register, ID: DRKS00021499.

\section{Clinical outcomes}

As the primary endpoint, the DBT group's interaction effects were tested against those of the control group and defined as having statistically better performance in at least two neuropsychological tests three months after surgery compared to baseline testing. The primary outcome measure were the results of the neuropsychological test battery. We assumed better neuropsychological outcomes in the DBT group, as previous cardiosurgical studies have shown the effectiveness of DBT filters in reducing microemboli $(11,19)$. As secondary endpoints, we assessed the impact of PEA surgery on symptoms of depression and anxiety at follow-up, as well as the extent of acute ischemic brain lesions in the entire sample and between the groups.

\section{Sample size estimation}

According to own study results using the DBT (11), we assumed a medium-size effect with respect to the primary hypothesis. On basis of $80 \%$ power $(\alpha=0.05$, one-tailed) and an effect magnitude of Cohen's $d=0.6$, the inclusion of 36 patients per group was estimated as the optimal sample size. From our previous studies, we expected a dropout rate of $20 \%$ with respect to neuropsychological follow-up examination, so we planned to include 50 patients per study group.

\section{Surgery}

The aim of PEA surgery is to remove all obstructive fibrotic thromboembolic material from the pulmonary arteries. In all patients, the surgical procedure was performed under conditions of cardiopulmonary bypass and nonpulsatile flow. PEA was performed in accordance with standardized surgical and anesthetic methods (20). Using a median sternotomy, ECC was established and hypothermia $\left(\leq 20^{\circ} \mathrm{C}\right)$ was induced to facilitate the desobliteration of both pulmonary arteries during periods of deep hypothermic circulatory arrest.

\section{Neuropsychological assessment}

Neuropsychological testing was performed at baseline (1-2 days before surgery) and at 3 months ( \pm 2 weeks) postoperatively by an experienced psychologist using a battery of well-established tests. All neuropsychological test data have continuous metric niveau. The cognitive test battery assesses verbal and visual short- and long-term memory, attention and processing speed, cognitive flexibility and word fluency (Table 1). The allocation of single tests to the respective cognitive domains was based on the tests' content. Symptoms of depression and anxiety were assessed using the validated German version of the Hospital Anxiety and Depression Scale (HADS) (27). In all domains, parallel test forms were used if available to avoid learning effects from pre- to postoperative neuropsychological testing. 
Table 1 Allocation of neuropsychological tests and tasks to cognitive domains

\begin{tabular}{lll}
\hline Cognitive domain & Test & Task \\
\hline Visual memory & SKT Pictorial Memory (21) & Recalling objects immediately \\
& NVLT (22) & Recalling objects after delay \\
& & Recognize objects \\
& VLMT (23) & Recognize repeated symbols \\
Verbal memory & & Recalling items of word list (learning process) \\
& & Recalling words immediately \\
& & Recalling words after delay \\
Attention and processing speed & Digit symbol test (24) & Recognize words \\
& Number cancellation (25) & Drawing symbols in boxes below numbers \\
Cognitive flexibility & Cancelling target numbers in a choice list \\
Word fluency & RWT (26) & Naming an interfering letter (e.g., "S" instead of "T") \\
\hline
\end{tabular}

SKT, Syndrom-Kurztest; NVLT, non-verbal learning test; RWT, word fluency test; VLMT, Verbaler Lern- und Merkfähigkeitstest.

\section{Magnetic resonance imaging (MRI)}

Cranial MRI was performed 6 to 10 days after surgery using a 3-T scanner (Skyra; Siemens, Erlangen, Germany). The protocol included a $\mathrm{T} 2$-weighted turbo spin-echo sequence [slice thickness $=3 \mathrm{~mm}$, field of view $(\mathrm{FOV})=220 \times 220 \mathrm{~mm}^{2}$, matrix $=512 \times 391$, repetition time $(\mathrm{TR})=7.490 \mathrm{~ms}$, echo time (TE) $=100 \mathrm{~ms}$, a T2-weighted turbo spin-echo sequence for dark fluid (slice thickness $=3 \mathrm{~mm}, \mathrm{FOV}=220 \times 220 \mathrm{~mm}^{2}$, matrix $=320 \times 224, \mathrm{TR}=7.000 \mathrm{~ms}, \mathrm{TE}=81 \mathrm{~ms})$ a T1weighted FLASH sequence (slice thickness $=3 \mathrm{~mm}$, FOV $=220 \times 220 \mathrm{~mm}^{2}$, matrix $=320 \times 320, \mathrm{TR}=250 \mathrm{~ms}$, TE $=2.49 \mathrm{~ms}$ ), and a diffusion-weighted echo-planar imaging sequence (slice thickness $=3 \mathrm{~mm}, \mathrm{FOV}=220 \times 220 \mathrm{~mm}^{2}$, matrix $=160 \times 160, \mathrm{TR}=7.720 \mathrm{~ms}$, TE $=64 \mathrm{~ms}$, slice gradients of b-values $=0$ and $1,000 \mathrm{~s} / \mathrm{mm}^{2}$ ). Two blinded, experienced observers used the diffusion-weighted sequence for registration of acute ischemic lesions.

\section{Statistical analysis}

Group differences regarding patients' characteristics (demographics, baseline neuropsychological test results and intra- and postoperative data) were descriptively analyzed using chi-square tests, $t$-tests or Mann-Whitney-U-test depending on the respective variable's level of measurement.

For the primary outcome, the DBT group was tested against the control group using repeated analyses of variance (ANOVA) with groups as a between-subjects factor (DBT vs. control) and assessment time as within-subjects factor (baseline and 3-month follow-up assessment) for all cognitive tests. The secondary outcomes were analyzed as follows: the progress of symptoms of depression and anxiety were tested for group differences using repeated ANOVA. The incidence of acute brain lesions was examined in terms of the frequency between the groups using Pearson's chisquare test. Assumptions for repeated ANOVAs were tested using Levene test for homogeneity of variance. Normal distribution was tested using the Shapiro-Wilk test. However, the ANOVA is robust against violation of normal distribution (28). Additional covariates were included in the analysis to control for potential confounding effects related to age and gender. In variance analyses, the eta square $\left(\eta^{2}\right)$ was calculated as the effect size measure, with $\eta^{2} \geq 0.01$ defining a small effect, $\eta^{2} \geq 0.06$ a medium effect and $\eta^{2} \geq 0.14$ a large effect (29). All analyses were performed using SPSS software (SPSS, Chicago, IL, USA). The level of significance was set at 0.05 .

\section{Results}

Between April 2012 and March 2018, 764 PEA surgeries were performed at the Department of Cardiac Surgery of the Kerckhoff Heart and Thorax Centre in Bad Nauheim, Germany with 100 patients eligible for study participation. 


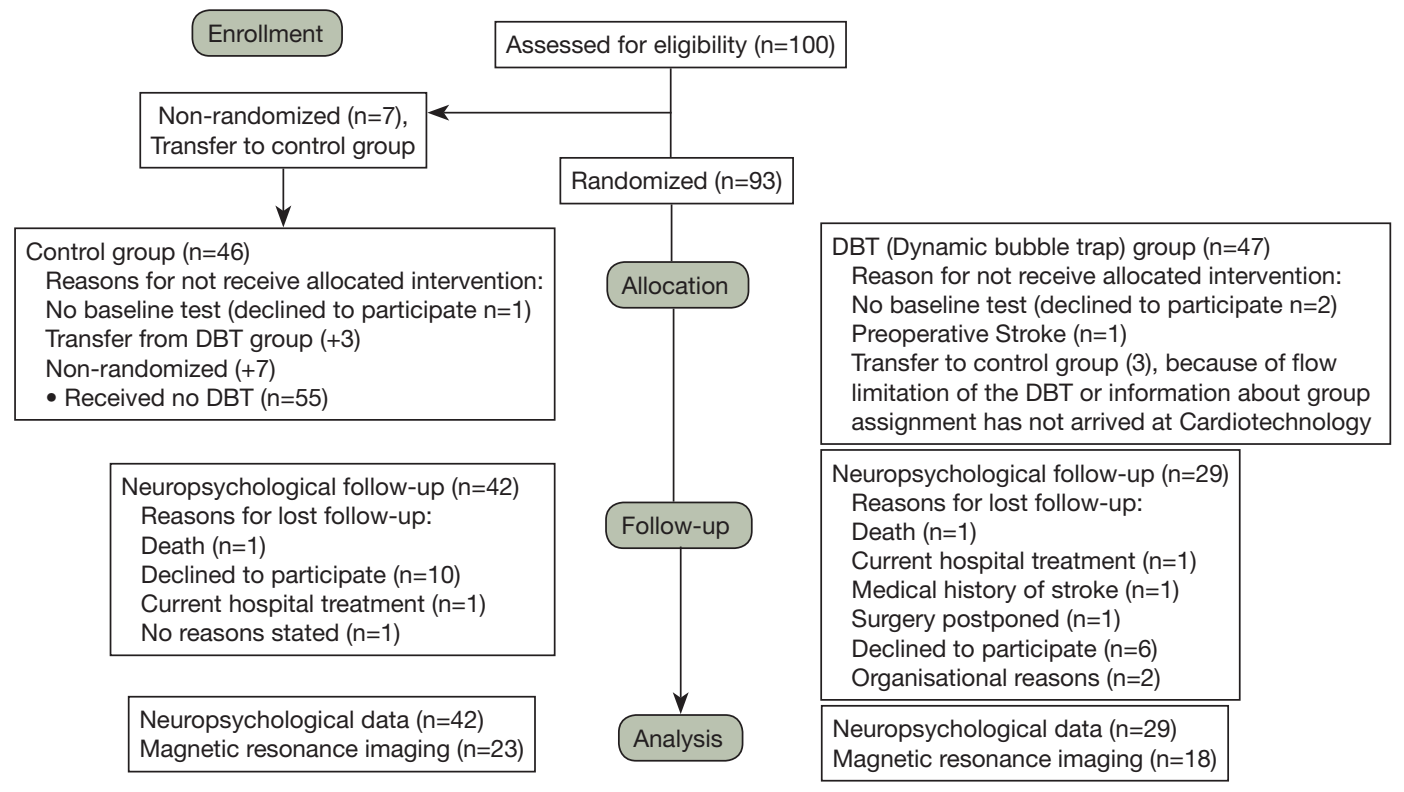

Figure 1 Consolidated Standards of Reporting Trials (CONSORT) flow chart illustrating all steps in the study from enrolment to group allocation and follow-up.

In total, 93 patients randomly were allocated to one of the two groups. Another seven patients were accidently not randomized. These patients were not excluded from the study but were assigned to the control group because they had already received neuropsychological testing and agreed to participate in the study. For another three patients, the DBT could not be used because the device has a flow limitation, which means that the DBT cannot be used in severely overweight patients. These three patients were therefore transferred to the control group. The decision on this was made by the perfusionist. To assess whether the groups differed from pre- to post-testing, 71 patients were included in the neuropsychological data analysis (29 in the DBT group, 42 in the control group). Magnetic resonance imaging (MRI) was performed on 41 patients (23 in the control group, 18 in the DBT group). The reasons for the imbalance in group size, and for dropouts and participants lost to follow-up, are shown in Figure 1.

\section{Patient characteristics and clinical parameters}

The patients' characteristics, surgical details and postoperative complications are shown in Table 2 and Table 3. The two groups differed only in terms of body mass index (BMI) $(\mathrm{P}=0.027)$; all other characteristics and clinical parameters were equally distributed. Furthermore, the occurrence of postoperative complications did not differ between the groups. The most common complication was supraventricular arrhythmia. Severe complications, such as multiorgan failure or reperfusion edema, were rare. Two patients, one from each group, died after surgery. One patient died from many postoperative complications, including reperfusion edema. Another patient died as a result of postoperative pneumonia. Another patient in the DBT group suffered a severe bihemispheric embolic stroke that required intensive care therapy, transfer to another stroke-specialized hospital and neurological rehabilitation immediately thereafter, so this patient was not included in the follow-up testing.

\section{Primary endpoint: Interaction effects of neuropsychological performance}

Table 4 shows the results of neuropsychological testing before the PEA and at the 3-month follow-up. At baseline, statistically homogenous performance in the two groups was found in all measures except the "Number cancellation test." In this subtest, which measures attention and processing speed, the control group achieved better results than the DBT group $[\mathrm{t}(69)=-2.03 ; \mathrm{P}=0.046]$. Across all tests, higher scores reflected better performance except for the "Number cancellation test" and the "SKT - 
Table 2 Study patients' baseline and clinical characteristics

\begin{tabular}{|c|c|c|c|}
\hline Clinical characteristics & DBT group & Control group & $P$ value \\
\hline Gender, n (\%) & Male: 24 (58.5); Female: 17 (41.5) & Male: 23 (45.1); Female: 28 (54.9) & 0.200 \\
\hline Education, mean $\pm S D$, years & $12.6 \pm 3.2$ & $11.7 \pm 4.1$ & 0.31 \\
\hline $\mathrm{BMI}$, mean $\pm \mathrm{SD}, \mathrm{kg} / \mathrm{m}^{2}$ & $26.8 \pm 4.0$ & $30.8 \pm 7.9$ & $0.027^{*}$ \\
\hline Arterial hypertension, n (\%) & $14(34.1)$ & $19(37.3)$ & 0.757 \\
\hline Coronary heart disease, n (\%) & $7(17.1)$ & $7(13.7)$ & 0.657 \\
\hline $\mathrm{mPAP}$, mean $\pm \mathrm{SD}, \mathrm{mmHg}$ & $38.82 \pm 12.71$ & $37.29 \pm 11.24$ & 0.55 \\
\hline $\mathrm{PVR}$, mean $\pm \mathrm{SD}, \mathrm{dyn} / \mathrm{s} / \mathrm{cm}^{2}$ & $544.44 \pm 268.11$ & $544.71 \pm 340.61$ & 0.99 \\
\hline
\end{tabular}

*, significant differences with $\mathrm{P}<0.05$. Renal insufficiency was defined by creatinine value above the in-house norms (man: $>1.2 \mathrm{mg} / \mathrm{dL}$, women: $>0.9 \mathrm{mg} / \mathrm{dL}$ ). DBT, dynamic bubble trap; BMI, body mass index; Mpap, mean pulmonary artery pressure; PVR, pulmonary vascular resistance.

Table 3 Study patients' surgical details, postoperative data and complications

\begin{tabular}{|c|c|c|c|}
\hline & DBT group & Control group & $P$ value \\
\hline \multicolumn{4}{|l|}{ Surgical details } \\
\hline Duration of surgery, mean $\pm \mathrm{SD}$, min & $430.7 \pm 63.0$ & $417.6 \pm 44.7$ & 0.289 \\
\hline Duration of circulatory arrest, mean $\pm \mathrm{SD}$, min & $35.2 \pm 10.4$ & $31.7 \pm 8.6$ & 0.085 \\
\hline Lowest body temperature, mean $\pm \mathrm{SD},{ }^{\circ} \mathrm{C}$ & $17.9 \pm 1.2$ & $17.7 \pm 1.2$ & 0.588 \\
\hline Duration of aortic clamping, mean $\pm \mathrm{SD}$, min & $106.2 \pm 35.8$ & $106.0 \pm 20.6$ & 0.732 \\
\hline \multicolumn{4}{|l|}{ Postoperative data } \\
\hline Duration of stay in ICU, mean \pm SD, days & $4.3 \pm 4.3$ & $2.6 \pm 1.3$ & 0.069 \\
\hline Duration of stay in ward, mean $\pm S D$, days & $14.3 \pm 4.6$ & $13.4 \pm 4.4$ & 0.331 \\
\hline Acute renal failure, $\mathrm{n}(\%)$ & $4(9.8)$ & $1(2.0)$ & 0.101 \\
\hline Infection, n (\%) & $5(9.8)$ & $5(12.2)$ & 0.714 \\
\hline Pericardial effusions, n (\%) & $2(4.9)$ & $7(13.7)$ & 0.156 \\
\hline Rethrombosis, n (\%) & 0 & $3(5.9)$ & 0.114 \\
\hline Multiorgan failure, n (\%) & $2(4.9)$ & 0 & 0.111 \\
\hline Reperfusion edema, n (\%) & $3(7.3)$ & $3(5.9)$ & 0.782 \\
\hline Stroke, n (\%) & $1(2.4)$ & 0 & 0.262 \\
\hline
\end{tabular}

DBT, dynamic bubble trap; ICU, intensive care unit. 
Table 4 Scores on neuropsychological tests at baseline and 3 months after surgery for the control group and DBT group

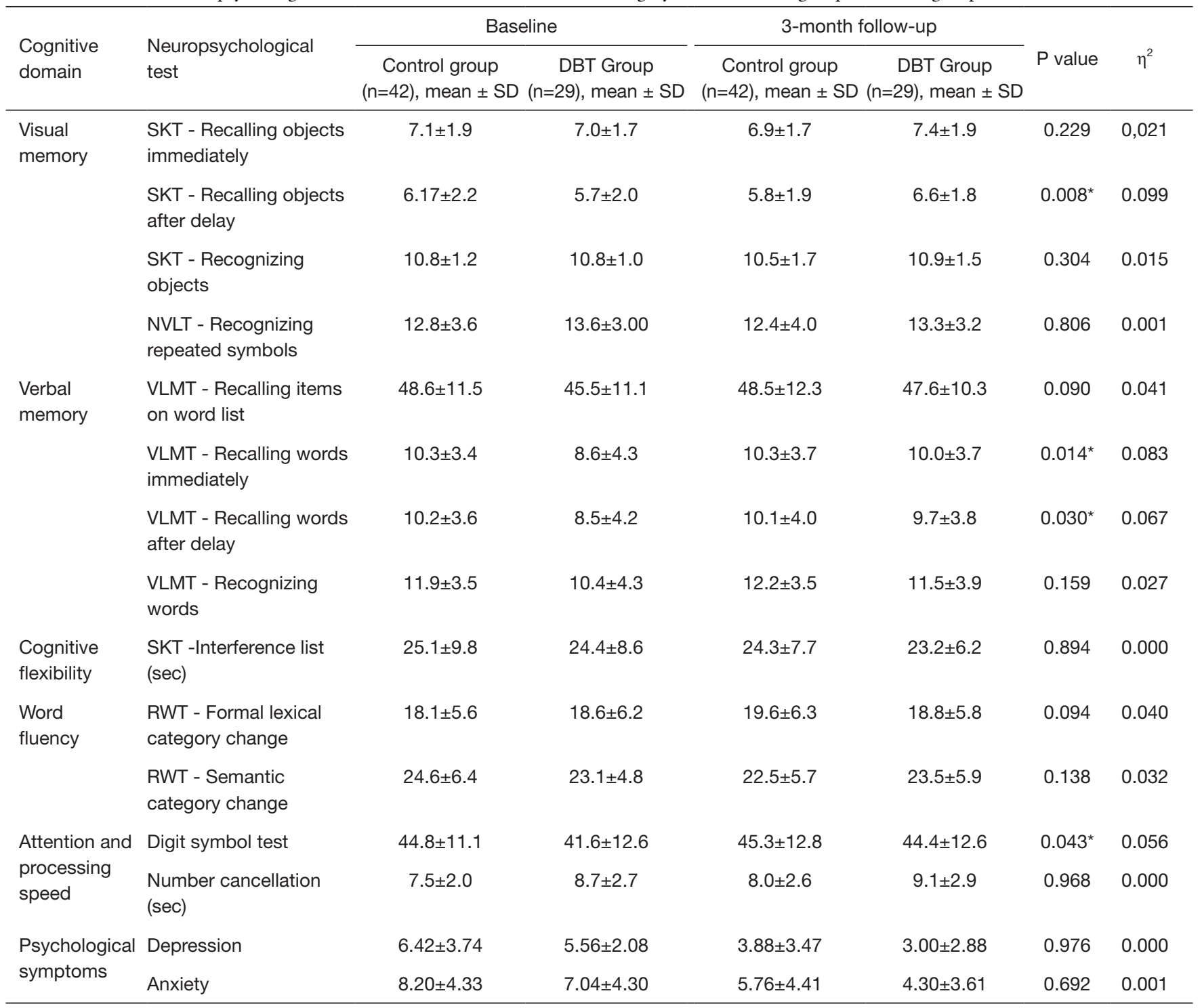

Higher test scores reflect better performance except for the "Number cancellation test" and the "SKT - Interference list". P values refers to the interactions effects of repeated analyses of variance (ANOVA). DBT, dynamic bubble trap; SKT, Syndrom-Kurztest; NVLT, non-verbal learning test; RWT, word fluency test; VLMT, Verbaler Lern- und Merkfähigkeitstest.

Interference list." When testing visual memory, the DBT group showed improved performance in visual longterm memory compared to the control group in "SKT - Recalling objects after delay" $[\mathrm{F}(1.67)=7.47, \mathrm{P}=0.008$, $\left.\eta^{2}=0.099\right]$. Significant group differences were also found in the domain of verbal memory. Patients in the DBT group exhibited improved performance compared to control group in "VLMT - Recalling words immediately" $[\mathrm{F}(1.67)$ $\left.=6.31, \mathrm{P}=0.014, \eta^{2}=0.083\right]$ and "VLMT - Recalling words after delay" $\left[\mathrm{F}(1,67)=4.89, \mathrm{P}=0.030, \eta^{2}=0.067\right]$. A significant group difference was also found in the cognitive domain of attention and processing speed. In the "digit symbol test", the DBT group showed a significantly improved performance compared to the control group $[\mathrm{F}(1.67)$ $\left.=4.256, P=0.043, \eta^{2}=0.056\right]$. The interaction effects for these tests are shown graphically in Figure 2. For all other interaction effects, no significant changes between pre- and postoperative neuropsychological testing could be observed. 

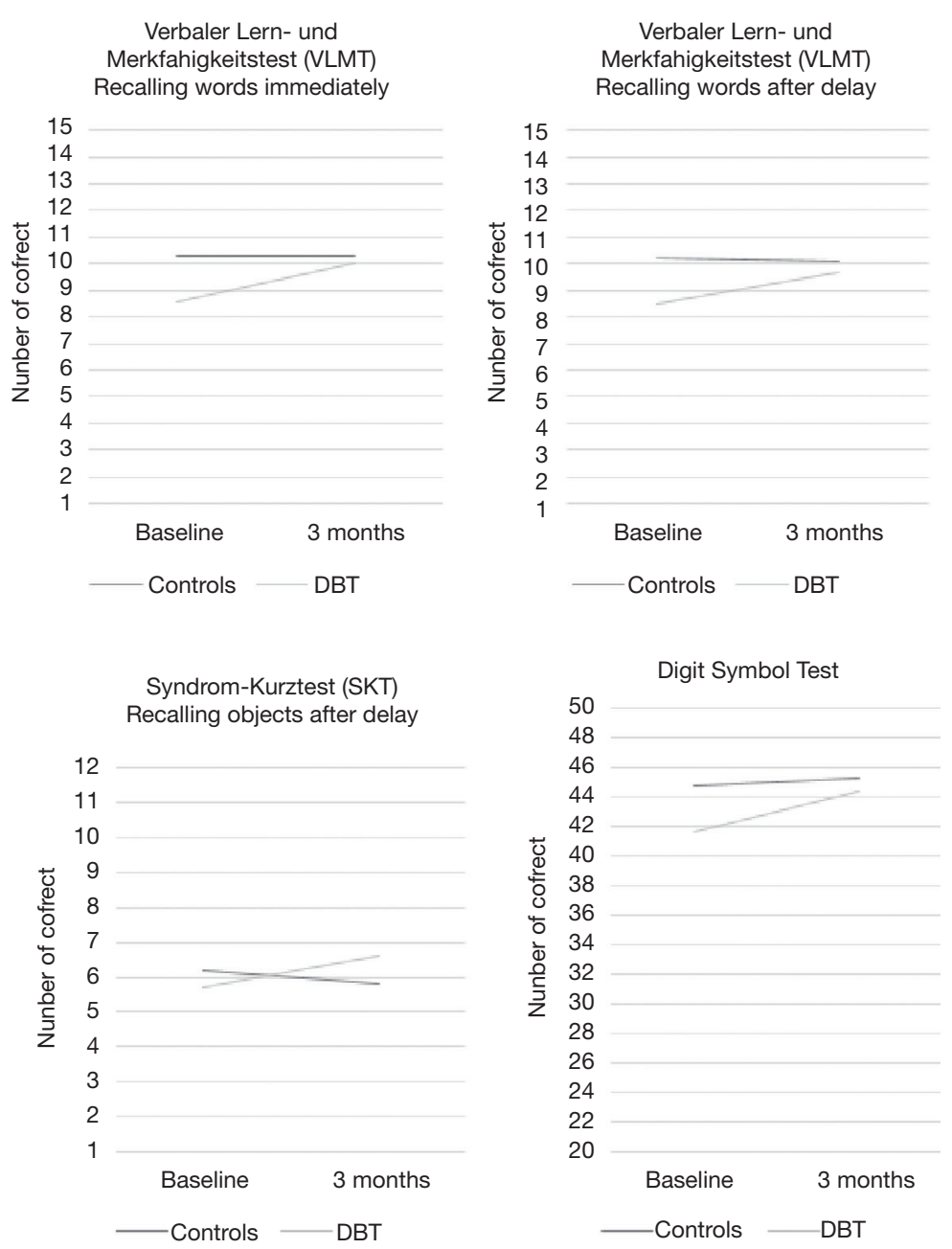

Figure 2 Interaction effects of neuropsychological functions between the DBT (dynamic bubble trap) group and control group. The mean values for the number of correct items at the baseline and 3-month follow-up assessment for SKT (Syndrom-Kurztest), VLMT (Verbaler Lern- und Merkfähigkeitstest) and Digit Symbol Test for the two investigated groups are given. A higher test value indicates better test performance. The figure shows that the DBT group improved to a greater extent compared to the control group. While the performance in the control group remained almost identical, there was an improved test performance in the DBT group compared to baseline testing.

\section{Secondary outcomes}

\section{Depression and anxiety scores}

In baseline testing, anxiety and depression scores did not differ significantly between the groups. There were also no significant differences in depression and anxiety scores between the groups (interaction) in follow-up testing (see Table 4). When examined across the entire sample, symptoms of depression and anxiety improved significantly after surgery $\left[\mathrm{F}(1.67)=57.50, \mathrm{P}<0.001, \eta^{2}=0.462 ; \mathrm{F}(1.67)=\right.$ 48.05, $\mathrm{P}<0.001, \eta^{2}=0.418$, respectively].

\section{Acute ischemic brain lesions}

MRI was performed on 41 patients (44.6\%). An acute ischemic brain lesion was detected in 2 out of 18 patients in the DBT group (11\%). One of these patients suffered a clinically silent micro-infarction (i.e., a neurological examination revealed no focal deficit, and the patient negated subjective symptoms). The other patient suffered a severe bihemispheric embolic stroke that required intensive care therapy. In the control group, none of the 23 patients who underwent MRI suffered acute brain micro-lesions. 


\section{Discussion}

PEA is marked by a long duration of ECC with a probably high load of microembolization, which may have a negative impact on cognitive function. To our knowledge, this is the first study comparing the effectiveness of the DBT device with standard care on cognitive function and ischemic micro-lesions in patients undergoing PEA.

The main findings of the study are as follows: (I) in the DBT group, neuropsychological test performances improved in terms of visual and verbal memory compared to the control group 3 months postoperatively, (II) symptoms of depression and anxiety improved significantly after surgery independent of group allocation, and (III) no group differences regarding the incidence of micro-infarctions were detected in MRI.

The study's results support the use of an intraoperative filter that reduces gaseous microemboli during PEA with ECC. Studies in cardiac surgery have already demonstrated that DBT use effectively eliminates gaseous microemboli and that this elimination can improve cognitive function $(11,19,30)$. Although other studies have already shown an improvement in cognitive performance after PEA $(8,9)$, our data indicate that the targeted reduction of gaseous microembolism leads to a greater improvement in neuropsychological performance. This result demonstrates the essential role of gaseous embolism in the development of postoperative cognitive deficits.

The reasons for the improved neuropsychological performance after PEA, as reported in previous studies, seem to be various, but it can be hypothesized that these positive changes can be mainly explained by the improved oxygen supply and hemodynamic situation, as these factors may contribute to increased cognitive performance. Patients' awareness of having survived a complex surgical procedure intended to treat a life-threatening disease, accompanied by an enhanced quality of life (31) and increased social activity are further reasons that may contribute to increased cognitive performance. In addition, postoperative depression and anxiety scores improved, potentially contributing to ameliorated cognition.

Because of the imbalance in group sizes due to various reasons of cross over, a sensitivity analysis was performed in which four subjects from the control group were removed from the analysis. No follow-up testing was available for the other patients who were mistakenly not randomized or transferred to the control group because of a high BMI. The sensitivity analysis also showed that the statistical effects of improved neuropsychological performance remained statistically significant in the DBT group compared to the control group.

It is notable that the ICU stay was longer in the control group compared to the DBT group. Long ICU stay may increase the risk for hypoactive delirium which is difficult to diagnose correctly and may be overlooked. Delirium may affect cognitive function. The presence of delirium, both hyperactive and hypoactive, was evaluated on the intensive care unit. If delirium was suspected, the department of psychocardiology was involved to confirm the diagnosis after a thorough clinical evaluation. Accordingly, we do not assume that patients with delirium were undiagnosed. The incidence of delirium did not differ statistically between groups. Therefore, the 3 -month outcome should be independent of the presence of delirium.

So far, the rate of peri-interventional ischemic stroke during PEA has not been assessed systematically. In a systematic review, Patel et al. reported acute cerebral ischemic lesions detected by DWI MRI in $15-61 \%$ of patients following on-pump cardiac surgery (32). Compared to these data, the frequency of acute cerebral ischemia after PEA in our cohort was considerably lower: one patient suffered a severe embolic stroke, and another patient displayed a small asymptomatic ischemic lesion on MRI. It has already been shown that patient age, the presence of subacute ischemic lesions and leukoencephalopathy, as well as atherosclerosis of the proximal aorta and the aortic arch, increase the risk of intraoperative ischemic lesions during ECC $(33,34)$. These are mainly age-related conditions that are less present in the younger population observed here, which might partially explain the lower stroke rate.

\section{Strengths and limitations}

The strength of this study is that it is a randomized, controlled trial in which the investigators were blinded to the outcome of the randomization. In addition, the PEA was performed by only two experienced surgeons.

A first and major limitation of the study is the imbalance in its group sizes resulting from the fact that seven patients were allocated to the control group without randomization. Another three patients initially randomized to the DBT group had to be transferred to the control group because DBT use was not possible in these severely overweight patients due to flow limitations of the DBT. A high BMI should be considered as an exclusion criterion in future studies. 
In addition, only 41 patients received an MRI. In some cases, patients refused examination, or MRI could not be performed for medical or organizational reasons. Furthermore, no long-term effects were assessed in this study.

\section{Conclusions}

Our results provide support that intraoperative DBT use has a positive influence on neuropsychological outcomes after PEA. This effect is most likely caused by the reduction of gaseous microemboli by the use of the DBT. This study's results justify the consideration whether DBT can be used in all patients undergoing PEA in the future.

\section{Acknowledgments}

Funding: This work was supported by the Willy- and Monika-Pitzer Foundation, Bad Nauheim, Germany.

\section{Footnote}

Reporting Checklist: The authors have completed the CONSORT reporting checklist. Available at https://dx.doi. org/10.21037/jtd-21-831

Trial Protocol: Available at https://dx.doi.org/10.21037/jtd21-831

Data Sharing Statement: Available at https://dx.doi. org/10.21037/jtd-21-831

Conflicts of Interest: All authors have completed the ICMJE uniform disclosure form (available at https://dx.doi. org/10.21037/jtd-21-831). The following authors report financial activities outside the submitted work: Christoph B. Wiedenroth reports having received lecture honorary from Actelion, Bayer, BTG, MSD and Pfizer. Stefan Guth reports having received lecture honorary from Actelion, Bayer, MSD and Pfizer. The other authors have no conflicts of interest to declare.

Ethical Statement: The authors are accountable for all aspects of the work in ensuring that questions related to the accuracy or integrity of any part of the work are appropriately investigated and resolved. The trial was conducted in accordance with the Declaration of Helsinki (as revised in 2013). The study was approved by the ethics committee of Justus-Liebig University Giessen (AZ 157/11) and informed consent was taken from all individual participants.

Open Access Statement: This is an Open Access article distributed in accordance with the Creative Commons Attribution-NonCommercial-NoDerivs 4.0 International License (CC BY-NC-ND 4.0), which permits the noncommercial replication and distribution of the article with the strict proviso that no changes or edits are made and the original work is properly cited (including links to both the formal publication through the relevant DOI and the license). See: https://creativecommons.org/licenses/by-nc-nd/4.0/.

\section{References}

1. Galiè N, Hoeper MM, Humbert M, et al. Guidelines for the diagnosis and treatment of pulmonary hypertension: the Task Force for the Diagnosis and Treatment of Pulmonary Hypertension of the European Society of Cardiology (ESC) and the European Respiratory Society (ERS), endorsed by the International Society of Heart and Lung Transplantation (ISHLT). Eur Heart J 2009;30:2493-537.

2. Konstantinides SV, Torbicki A, Agnelli G, et al. 2014 ESC guidelines on the diagnosis and management of acute pulmonary embolism. Eur Heart J 2014;35:3033-69, 3069a-3069k.

3. Bazmpani MA, Arvanitaki A, Toumpourleka M, et al. Epidemiology and management of chronic thromboembolic pulmonary hypertension: experience from two expert centers. Hellenic J Cardiol 2018;59:16-23.

4. Wilkens H, Konstantinides S, Lang IM, et al. Chronic thromboembolic pulmonary hypertension (CTEPH): Updated Recommendations from the Cologne Consensus Conference 2018. Int J Cardiol 2018;272S:69-78.

5. 2015 ESC/ERS Guidelines for the diagnosis and treatment of pulmonary hypertension. Eur Heart J 2016;37:67-119.

6. Delcroix M, Torbicki A, Gopalan D, et al. ERS statement on chronic thromboembolic pulmonary hypertension. Eur Respir J 2021;57:2002828.

7. Hsieh WC, Jansa P, Huang WC, et al. Residual pulmonary hypertension after pulmonary endarterectomy: A metaanalysis. J Thorac Cardiovasc Surg 2018;156:1275-87.

8. Vanini B, Grazioli V, Sciortino A, et al. Neuropsychological outcomes after pulmonary endarterectomy using moderate hypothermia and periodic circulatory arrest. J Heart Lung Transplant 2018;37:860-4. 
9. Vuylsteke A, Sharples L, Charman G, et al. Circulatory arrest versus cerebral perfusion during pulmonary endarterectomy surgery (PEACOG): a randomised controlled trial. Lancet 2011;378:1379-87.

10. Boeken U, Litmathe J, Feindt P, et al. Neurological complications after cardiac surgery: risk factors and correlation to the surgical procedure. Thorac Cardiovasc Surg 2005;53:33-6.

11. Gerriets T, Schwarz N, Sammer G, et al. Protecting the brain from gaseous and solid micro-emboli during coronary artery bypass grafting: a randomized controlled trial. Eur Heart J 2010;31:360-8.

12. Schoenburg M, Kraus B, Muehling A, et al. The dynamic air bubble trap reduces cerebral microembolism during cardiopulmonary bypass. J Thorac Cardiovasc Surg 2003;126:1455-60.

13. Evered L, Scott DA, Silbert B, et al. Postoperative cognitive dysfunction is independent of type of surgery and anesthetic. Anesth Analg 2011;112:1179-85.

14. Knipp SC, Matatko N, Wilhelm H, et al. Cognitive outcomes three years after coronary artery bypass surgery: relation to diffusion-weighted magnetic resonance imaging. Ann Thorac Surg 2008;85:872-9.

15. Newman MF, Kirchner JL, Phillips-Bute B, et al. Longitudinal assessment of neurocognitive function after coronary-artery bypass surgery. N Engl J Med 2001;344:395-402.

16. Newman MF, Grocott HP, Mathew JP, et al. Report of the substudy assessing the impact of neurocognitive function on quality of life 5 years after cardiac surgery. Stroke 2001;32:2874-81.

17. Phillips-Bute B, Mathew JP, Blumenthal JA, et al. Association of neurocognitive function and quality of life 1 year after coronary artery bypass graft (CABG) surgery. Psychosom Med 2006;68:369-75.

18. Steinmetz J, Christensen KB, Lund T, et al. Long-term consequences of postoperative cognitive dysfunction. Anesthesiology 2009;110:548-55.

19. Schönburg M, Ziegelhoeffer T, Kraus B, et al. Reduction of gaseous microembolism during aortic valve replacement using a dynamic bubble trap. Gen Physiol Biophys 2006;25:207-14.

20. Guth S, Wiedenroth CB, Kramm T, et al. Pulmonary endarterectomy for the treatment of chronic thromboembolic pulmonary hypertension. Expert Rev Respir Med 2016;10:673-84.

21. Lehfeld H, Erzigkeit H. The SKT--a short cognitive performance test for assessing deficits of memory and attention. Int Psychogeriatr 1997;9 Suppl 1:115-21.

22. Sturm W, Willmes K. Verbaler Lerntest, Nonverbaler Lerntest:(VLT/NVLT). Göttingen: Hogrefe, 1999.

23. Helmstaedter C, Lendt M, Lux S. VLMT: Verbaler Lernund Merkfähigkeitstest. Beltz Test, 2001.

24. Spreen O, Strauss E. A Compendium of Neuropsychological Tests: Administration, Norms and Commentary. Oxford University Press, 1991.

25. Brickenkamp R, Schmidt-Atzert L, Liepmann D. Test d2-Revision (d2-R). Göttingen: Hogrefe, 2009.

26. Aschenbrenner S, Tucha O, Lange K. Manual zum RWT (Regensburger Wortflüssigkeits-Test; Handanweisung). Hogrefe Verlag für Psychologie, Göttingen, Bern, Toronto, Seattle 2000.

27. Petermann F. Hospital anxiety and depression scale, deutsche version (HADS-D). Zeitschrift für Psychiatrie, Psychologie und Psychotherapie, 2015.

28. Blanca MJ, Alarcón R, Arnau J, et al. Non-normal data: Is ANOVA still a valid option? Psicothema 2017;29:552-7.

29. Cohen J. Statistical power analysis for the behavioral sciences: Routledge; 2013.

30. Perthel M, Kseibi S, Bendisch A, et al. Use of a dynamic bubble trap in the arterial line reduces microbubbles during cardiopulmonary bypass and microembolic signals in the middle cerebral artery. Perfusion 2005;20:151-6.

31. Nadelson MR, Sanders RD, Avidan MS. Perioperative cognitive trajectory in adults. Br J Anaesth 2014;112:440-51.

32. Patel N, Minhas JS, Chung EM. The Presence of New MRI Lesions and Cognitive Decline After Cardiac Surgery: A Systematic Review. J Card Surg 2015;30:808-12.

33. Stolz E, Gerriets T, Kluge A, et al. Diffusion-weighted magnetic resonance imaging and neurobiochemical markers after aortic valve replacement: implications for future neuroprotective trials? Stroke 2004;35:888-92.

34. Djaiani G, Fedorko L, Borger M, et al. Mild to moderate atheromatous disease of the thoracic aorta and new ischemic brain lesions after conventional coronary artery bypass graft surgery. Stroke 2004;35:e356-8.

Cite this article as: El Shazly J, Gerriets T, Hennig J, Butz M, Kastaun S, Wiedenroth CB, Schoenburg M, Wollenschlaeger M, Bachmann G, Guth S, Juenemann M. Neuroprotective effects of dynamic bubble trap use in patients undergoing pulmonary endarterectomy: a two-arm randomized controlled trial. J Thorac Dis 2021;13(10):5807-5817. doi: 10.21037/jtd-21-831 